\title{
Metachronous bilateral mucoepidermoid carcinoma of the parotid gland
}

\author{
John C. Muthusami · Pranay Gaikwad · John P. Raj · Deepak T. Abraham - Meera Thomas · \\ Veracious Cornerstone
}

\begin{abstract}
We report a very rare case of bilateral mucoepidermoid carcinoma of the parotid gland that underwent bilateral parotidectomy with neck dissections and radiotherapy. This case has done well for three years and suggests that metachronous bilateral mucoepidermoid carcinoma of the parotid gland, if treated as per the merits of each side, has a reasonable survival.
\end{abstract}

Keywords Bilateral mucoepidermoid carcinoma - Parotid gland - Warthin's tumour
J. C. Muthusami - P. Gaikwad • J. P. Raj • D. T. Abraham •

M. Thomas • V. Cornerstone

Christian Medical College Hospital,

Vellore - 632 004,

Tamil Nadu, India

J. C. Muthusami ( $\square)$

e-mail: sur1@cmcvellore.ac.in

\section{Introduction}

Bilateral malignant tumours of the parotid gland are extremely rare. Warthin's tumour is the commonest bilateral neoplasm of the parotid gland. This is the first reported case of metachronous bilateral mucoepidermoid carcinomas arising de novo in the parotid glands. So far, only one previous case of asynchronous bilateral mucoepidermoid carcinoma arising in pre-existing bilateral warthin's tumors has been described.

\section{Gase report}

A 31-year-old man presented with bilateral parotid swellings of 4 years' duration. He was treated elsewhere with surgery and radiation for mucoepidermoid carcinoma in the left parotid 10 years ago. On the right side, there was a $6.5 \times 5.5 \times 3 \mathrm{~cm}$ smooth, firm swelling enclosed within the parotid fascia. On the left side, there was a Sistrunk's incision scar with skin changes due to radiation and a $4 \times 3 \times$ $2 \mathrm{~cm}$ firm swelling fixed to the skin and mastoid process. The facial nerve was normal on both sides.

Fine needle aspiration cytology taken from both the sides was reported as mucoepidermoid carcinoma. Ultrasonography of the recurrent side revealed an ill-defined heterogeneous focal lesion with posterior shadowing measuring $20 \times 10.2 \mathrm{~mm}$ and multiple nodes in the jugulo-digastric region. Metastatic evaluation was normal.

The patient underwent right radical parotidectomy with neck dissection. Intra-operatively, the tumour involved both superficial and deep lobes of the right parotid gland, encasing the facial nerve, infiltrating the masseter and the pterygoids. Multiple lymph nodes at levels II and III were noted. Three weeks later, the patient underwent left total conservative parotidectomy with neck dissection. Operative findings revealed a hard tumour fixed to the mastoid process separate from the main bulk of the gland with nodes at levels II and III. Postoperatively, the patient received external 
beam radiation therapy with $14 \mathrm{MeV}$ electrons on the right side. Histopathological examination of the surgical specimens stained with Haematoxylin and Eosin revealed both salivary glands infiltrated by tumour composed of closely packed dilated cysts lined by uni-, or multi- layered mucin producing tall columnar cells enclosing mucinous material. These cells exhibited mild to moderate nuclear anaplasia and occasional mitoses $(<4 / 10 \mathrm{HPF})$. Adjacent solid areas with islands of squamous and intermediate cells were seen ( $>20 \%$ of the tumour). The stroma showed extravasation of mucin (Fig. 1 Mucoepidermoid Carcinoma, left parotid gland; Fig. 2 Mucoepidermoid Carcinoma, right parotid gland). There was no perineural invasion. The tumour involved the marked deep resection margin on the right side. Histopathologically, the tumours were reported as intermediate grade mucoepidermoid carcinoma. Secondary tumour was seen in three intra-parotid nodes on the left side. All the resected lymph nodes on both sides of the neck were free of tumour.

\section{Discussion}

Mucoepidermoid carcinoma is the commonest malignant tumor of the parotid gland. The incidence of bilateral neoplasms of the parotid gland in a series of 1,805 cases was reported as $1.3 \%$, of which $86 \%$ were warthin's tumors [1]. A review of English literature underscores the rarity of bilateral malignant tumors of the parotid gland with only 15 cases reported so far [2]. One previous case had asynchronous bilateral mucoepidermoid carcinoma arising in pre-existing bilateral warthin's tumors [3].

Lymphatic drainage of the parotid gland occurs through cervical nodes and since there is no documented direct connection between the two sides, a parotid gland tumor on one side cannot be considered to be a metastasis from the other side [2].

The treatment for low-grade mucoepidermoid carcinoma affecting the superficial portion of the parotid gland is a superficial parotidectomy. For all other lesions, more radical ablative surgery of primary tumor is recommended with resection of facial nerve and related lymphatic system when affected [4]. Postoperative radiation therapy with high energy electrons augments surgical resection for high-grade neoplasms or when margins are close or involved [5]. A long-term follow-up is recommended since distant metastases can be found even decades after surgery [4].

Our patient presented with metachronous bilateral mucoepidermoid carcinoma of the parotid gland the left being recurrent. He underwent radical parotidectomy on the right side and total conservative parotidectomy on the left with neck dissections on both sides. Based on histopathological examination report, the patient received postoperative external beam radiation therapy on right side taking into

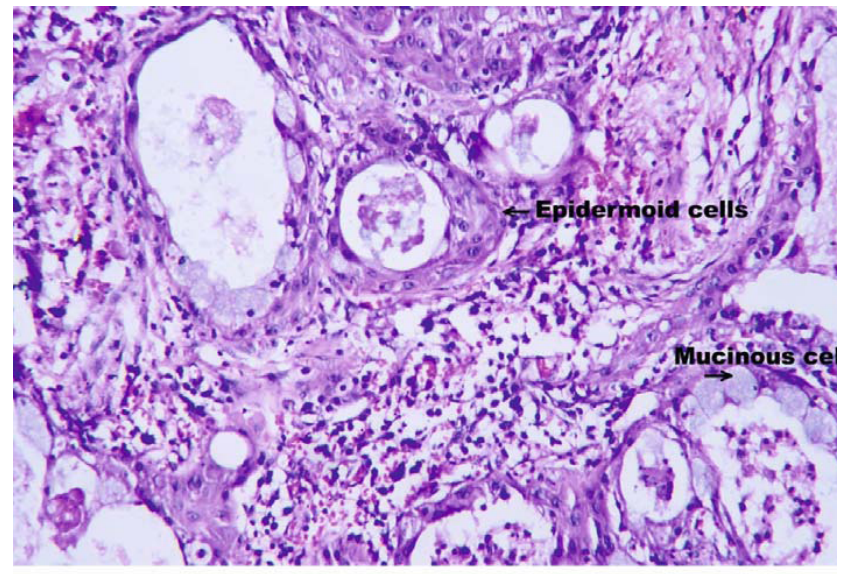

Mucoepidermoid carcinoma, left parotid gland (H \& E 400X)

Fig. 1 Mucoepidermoid Carcinoma, left parotid gland

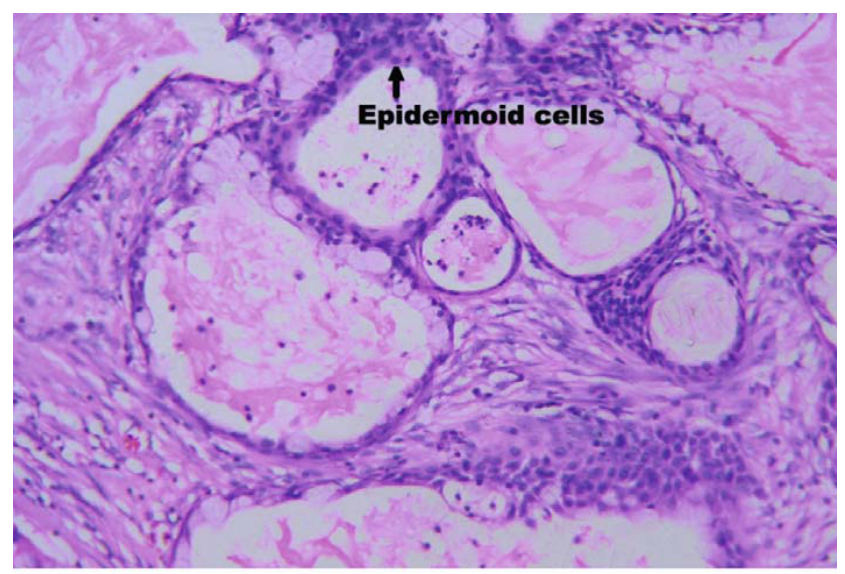

Mucoepidermoid carcinoma in right parotid gland.

(H\& E 400X)

Fig. 2 Mucoepidermoid Carcinoma, right parotid gland

account the radiation received on the left side previously. This is the first reported case of metachronous bilateral mucoepidermoid carcinomas, with no pre-existing tumor, arising in the parotid gland. The patient is doing well at 3 years.

It may be concluded that metachronous bilateral mucoepidermoid carcinoma of the parotid gland is the rarest of the rare and that it can be treated as per the merits of each side notwithstanding the awaited long-term outcome.

\section{References}

1. Turnbull AD, Frazell EL (1969) Multiple tumours of the major salivary glands. Am J Surg 118:787-789

2. Hakuba N, Hyodo M (2003) Synchronous bilateral mucoepidermoid carcinoma of the parotid gland. J laryngol otol 117(5):419-421 
Indian J. Otolaryngol. Head Neck Surg.

3. Assor D (1974) Bilateral Carcinoma of the parotid, one cancer arising in a Warthin's tumour. Am J Chin Pathol 61:270-274

4. Plambeck K, Friedrich RE, Schmelzle R (1996) Mucoepidermoid carcinoma of salivary gland origin. Classification, clinical pathological correlation, treatment results and long term follow up in 55 patients. J Cranio maxillofac surg 24 (3):133-139

5. Garden AS, el-Naggar AK, Morrison WH, et al (1997) Postoperative radiotherapy for malignant tumours of parotid gland. Int J Radiat Oncol Biol Phys 37(1):79-85 